\title{
A SINGULAR INITIAL VALUE PROBLEM FOR SOME FUNCTIONAL DIFFERENTIAL EQUATIONS
}

\author{
RAVI P. AGARWAL, DONAL O’REGAN, AND OLEKSANDR E. ZERNOV
}

Received 3 May 2004 and in revised form 13 May 2004

For the initial value problem $t^{r} x^{\prime}(t)=a t+b_{1} x(t)+b_{2} x\left(q_{1} t\right)+b_{3} t^{r} x^{\prime}\left(q_{2} t\right)+\varphi(t, x(t)$, $\left.x\left(q_{1} t\right), x^{\prime}(t), x^{\prime}\left(q_{2} t\right)\right), x(0)=0$, where $r>1,0<q_{i} \leqslant 1, i \in\{1,2\}$, we find a nonempty set of continuously differentiable solutions $x:(0, \rho] \rightarrow \mathbb{R}$, each of which possesses nice asymptotic properties when $t \rightarrow+0$.

\section{Introduction}

In this paper, we will consider singular initial value problems for a class of functional differential equations (FDEs). This problem has received very little attention in the literature to date; we refer the reader to $[2,3,5,7]$ where the question of solvability (in various spaces) and the number of solutions have been discussed. We remark that, even now, the asymptotic properties of solutions of FDEs are only partially understood. Our approach to this problem is essentially different from others known in the literature. In particular, we use qualitative methods $[1,7,8]$ together with standard fixed point theorems [6]. Our technique leads to the existence of continuously differentiable solutions with nice asymptotic properties.

\section{Main result}

Consider the initial value problem

$$
\begin{gathered}
t^{r} x^{\prime}(t)=a t+b_{1} x(t)+b_{2} x\left(q_{1} t\right)+b_{3} t^{r} x^{\prime}\left(q_{2} t\right) \\
+\varphi\left(t, x(t), x\left(q_{1} t\right), x^{\prime}(t), x^{\prime}\left(q_{2} t\right)\right), \\
x(0)=0,
\end{gathered}
$$

where $t \in(0, \tau)$ is a real variable, $x:(0, \tau) \rightarrow \mathbb{R}$ is a real unknown function, $r, a, b_{1}, b_{2}$, $b_{3}, q_{1}, q_{2}$ are constants, $r>1, b_{1} \neq 0,0<q_{i} \leqslant 1, i \in\{1,2\}$, and $\varphi: \mathscr{D} \rightarrow \mathbb{R}$ is a continuous 
function; here

$$
\begin{aligned}
\mathscr{D}=\{ & \left(t, y_{1}, y_{2}, y_{3}, y_{4}\right): t \in(0, \tau),\left|y_{1}\right|<\nu_{1} t, \\
& \left.\left|y_{2}\right|<v_{1} q_{1} t,\left|y_{3}\right|<v_{2} t^{1-r},\left|y_{4}\right|<v_{2}\left(q_{2} t\right)^{1-r}\right\} .
\end{aligned}
$$

In what follows, we will assume that the following conditions are satisfied:

$$
\begin{aligned}
& \left|\varphi\left(t, y_{1}, y_{2}, y_{3}, y_{4}\right)-\varphi\left(s, y_{1}, y_{2}, y_{3}, y_{4}\right)\right| \\
& \leqslant l_{1}(\mu)|t-s|, \quad\left(t, y_{1}, y_{2}, y_{3}, y_{4}\right) \in \mathscr{D},\left(s, y_{1}, y_{2}, y_{3}, y_{4}\right) \in \mathscr{D}, 0<\mu \leqslant t, 0<\mu \leqslant s, \\
& \left|\varphi\left(t, y_{1}, y_{2}, y_{3}, y_{4}\right)-\varphi\left(t, z_{1}, z_{2}, z_{3}, z_{4}\right)\right| \\
& \leqslant l_{2}(t)\left(\left|y_{1}-z_{1}\right|+\left|y_{2}-z_{2}\right|+t^{r}\left|y_{3}-z_{3}\right|+t^{r}\left|y_{4}-z_{4}\right|\right), \\
& \left(t, y_{1}, y_{2}, y_{3}, y_{4}\right) \in \mathscr{D},\left(t, z_{1}, z_{2}, z_{3}, z_{4}\right) \in \mathscr{D},
\end{aligned}
$$

where $l_{1}:(0, \tau) \rightarrow(0,+\infty)$ is a continuous nonincreasing function, $l_{2}:(0, \tau) \rightarrow(0,+\infty)$ is a continuous nondecreasing function, $\lim _{t \rightarrow+0} l_{2}(t)=0$, and

$$
\begin{gathered}
|a|<v_{1}\left|b_{1}+b_{2} q_{1}\right|, \\
q_{1}\left|b_{2}\right|<\left|b_{1}\right|\left(1-2\left|b_{3}\right| q_{2}^{1-r}\right) .
\end{gathered}
$$

Further, we will assume that

$$
\left|\varphi\left(t, c t, c q_{1} t, c, c\right)\right| \leqslant t \beta(t), \quad t \in(0, \tau),
$$

where $c=-a\left(b_{1}+b_{2} q_{1}\right)^{-1}, \beta:(0, \tau) \rightarrow(0,+\infty)$ is a continuously differentiable function and, moreover,

$$
\begin{gathered}
\lim _{t \rightarrow+0} \beta(t)=0, \quad \lim _{t \rightarrow+0} t^{r-1}(\beta(t))^{-1}=L_{1}, \quad \lim _{t \rightarrow+0} t \beta^{\prime}(t)(\beta(t))^{-1}=L_{2}, \\
0 \leqslant L_{i}<+\infty, \quad i \in\{1,2\} .
\end{gathered}
$$

Definition 2.1. For any $\rho \in(0, \tau)$, a continuously differentiable function $x:(0, \rho] \rightarrow \mathbb{R}$ is said to be $\rho$-solution of problem (2.1), (2.2) if

(1) $\left(t, x(t), x\left(q_{1} t\right), x^{\prime}(t), x^{\prime}\left(q_{2} t\right)\right) \in \mathscr{D}$ for all $t \in(0, \rho]$;

(2) $x$ identically satisfies the differential equation (2.1) for all $t \in(0, \rho]$.

We denote by $\mathcal{u}(\rho, M)$ the set of all continuously differentiable functions $u:(0, \rho] \rightarrow \mathbb{R}$ such that $|u(t)-c t| \leqslant M t \beta(t), t \in(0, \rho]$; here $\rho, M$ are (positive) constants, $\rho<\tau$.

The main result of this paper is the following theorem.

Theorem 2.2. There exist constants $\rho$, $M$ such that

(a) if $b_{1}>0$, then problem (2.1), (2.2) possesses an infinite set of $\rho$-solutions $x:(0, \rho] \rightarrow$ $\mathbb{R}$, each of which belongs to $U(\rho, M)$. Moreover, for any constant $\alpha$ which satisfies the condition $|\alpha-c \rho|<M \rho \beta(\rho)$, there exists at least one $\rho$-solution $x_{\alpha} \in \mathcal{U}(\rho, M)$ such that $x_{\alpha}(\rho)=\alpha$; 
(b) if $b_{1}<0$, then problem (2.1), (2.2) possesses at least one $\rho$-solution $x:(0, \rho] \rightarrow \mathbb{R}$ which belongs to $\mathcal{U}(\rho, M)$.

Proof. We can choose constants $Q$ and $M$ (cf. (2.6)) so that

$$
\begin{gathered}
2\left|b_{1}\right|<Q<q_{2}^{r-1}\left|b_{3}\right|^{-1}\left(\left|b_{1}\right|-q_{1}\left|b_{2}\right|\right) \\
M>\left(\left|\left(b_{3}-1\right) c\right| L_{1}+1\right)\left(\left|b_{1}\right|-q_{1}\left|b_{2}\right|-Q\left|b_{3}\right| q_{2}^{1-r}\right)^{-1} .
\end{gathered}
$$

Next, we choose $\rho$ such that

$$
0<\rho<\min _{1 \leqslant i \leqslant 9} \rho_{i}
$$

where all the $\rho_{i}$ are defined below (we choose $\rho$ small enough, $M$ and $Q$ large enough so that the choice of $Q, M$, and $\rho$ ensures the validity of all the reasoning given below).

Let $\mathscr{B}$ be the space of continuously differentiable functions $x:[0, \rho] \rightarrow \mathbb{R}$ with the norm

$$
\|x\|_{\Re}=\max _{t \in[0, \rho]}\left(|x(t)|+\left|x^{\prime}(t)\right|\right) .
$$

Let $\mathcal{U} \subseteq \mathscr{B}$ be such that for every element $u \in \mathcal{U}, u:[0, \rho] \rightarrow \mathbb{R}$ satisfies the inequalities

$$
\begin{gathered}
\left|u(t)-c t^{r+1}\right| \leqslant M t^{r+1} \beta(t), \\
\left|u^{\prime}(t)-(r+1) c t^{r}\right| \leqslant Q M t \beta(t), \quad t \in(0, \rho],
\end{gathered}
$$

and $u(0)=0, u^{\prime}(0)=0$; moreover,

$$
\forall \varepsilon>0, \forall u \in \mathcal{U}, \forall t_{i} \in[0, \rho], \quad i \in\{1,2\}:\left|t_{1}-t_{2}\right| \leqslant \delta(\varepsilon) \Longrightarrow\left|u^{\prime}\left(t_{1}\right)-u^{\prime}\left(t_{2}\right)\right| \leqslant \varepsilon,
$$

where $\delta(\varepsilon)=\varepsilon\left(8 B\left(t_{\varepsilon}\right)\right)^{-1}$; here $B\left(t_{\varepsilon}\right)=l_{1}\left(t_{\varepsilon}\right)+t_{\varepsilon}^{-r}, t_{\varepsilon}$ is a constant such that $t_{\varepsilon} \in(0, \rho)$, and if $t \in\left(0, t_{\varepsilon}\right]$, then the following inequalities are satisfied:

$$
Q M t \beta(t) \leqslant \frac{\varepsilon}{33}, \quad(1+r)|c| t^{r} \leqslant \frac{\varepsilon}{33} .
$$

It is easy to see that $U$ is a closed, bounded, and convex set. Moreover, $\mathcal{U}$ is a compact set (use the Arzelá theorem).

There exists a (small enough) $\rho_{1}>0$ such that if $\rho<\rho_{1}$, then

$$
\left(t, u(t) t^{-r}, u\left(q_{1} t\right)\left(q_{1} t\right)^{-r}, u^{\prime}(t) t^{-r}-r u(t) t^{-r-1}, u^{\prime}\left(q_{2} t\right)\left(q_{2} t\right)^{-r}-r u\left(q_{2} t\right)\left(q_{2} t\right)^{-r-1}\right) \in \mathscr{D}
$$

for all $u \in \mathcal{U}, t \in(0, \rho]$. 
Let $x=y t^{-r}$, where $y:(0, \tau) \rightarrow \mathbb{R}$ is a new unknown function. Then we obtain the following initial value problem:

$$
\begin{gathered}
y^{\prime}(t)=a t+b_{1} y(t) t^{-r}+r y(t) t^{-1}+b_{2} y\left(q_{1} t\right)\left(q_{1} t\right)^{-r} \\
+b_{3} q_{2}^{-r} y^{\prime}\left(q_{2} t\right)-r b_{3} q_{2}^{-r-1} t^{-1} y\left(q_{2} t\right) \\
+\varphi\left(t, y(t) t^{-r}, y\left(q_{1} t\right)\left(q_{1} t\right)^{-r}, y^{\prime}(t) t^{-r}-r y(t) t^{-r-1}\right. \\
\left.y^{\prime}\left(q_{2} t\right)\left(q_{2} t\right)^{-r}-r y\left(q_{2} t\right)\left(q_{2} t\right)^{-r-1}\right) \\
y(0)=0 .
\end{gathered}
$$

Now we will consider the initial value problem

$$
\begin{gathered}
y^{\prime}(t)=a t+b_{1} y(t) t^{-r}+r y(t) t^{-1}+b_{2} u\left(q_{1} t\right)\left(q_{1} t\right)^{-r} \\
+b_{3} q_{2}^{-r} u^{\prime}\left(q_{2} t\right)-r b_{3} q_{2}^{-r-1} t^{-1} u\left(q_{2} t\right) \\
+\varphi\left(t, u(t) t^{-r}, u\left(q_{1} t\right)\left(q_{1} t\right)^{-r}, u^{\prime}(t) t^{-r}\right. \\
\left.-r u(t) t^{-r-1}, u^{\prime}\left(q_{2} t\right)\left(q_{2} t\right)^{-r}-r u\left(q_{2} t\right)\left(q_{2} t\right)^{-r-1}\right), \\
y(0)=0,
\end{gathered}
$$

where $u \in \mathcal{U}$ is an arbitrary but fixed function. Let

$$
\mathscr{D}_{0}=\{(t, y): t \in(0, \rho], y \in \mathbb{R}\} \text {. }
$$

In $\mathscr{D}_{0}$, for (2.17), conditions for the existence and uniqueness theorem [4] and conditions for the continuous dependence of the initial data theorem [4] are fulfilled.

Now we denote

$$
\begin{aligned}
& \Phi_{1}=\left\{(t, y): t \in(0, \rho],\left|y-c t^{r+1}\right|=M t^{r+1} \beta(t)\right\}, \\
& \mathscr{D}_{1}=\left\{(t, y): t \in(0, \rho],\left|y-c t^{r+1}\right|<M t^{r+1} \beta(t)\right\}, \\
& H=\left\{(t, y): t=\rho,\left|y-c \rho^{r+1}\right|<M \rho^{r+1} \beta(\rho)\right\} .
\end{aligned}
$$

Let the function $A_{1}: \mathscr{D}_{0} \rightarrow[0,+\infty)$ be defined by the equality

$$
A_{1}(t, y)=\left(y-c t^{r+1}\right)^{2}\left(t^{r+1} \beta(t)\right)^{-2}
$$

and let $a_{1}: \mathscr{D}_{0} \rightarrow \mathbb{R}$ be the derivative of the function $A_{1}$. Using (2.17), we have

$$
\begin{aligned}
a_{1}(t, y)=2\left(t^{r+1} \beta(t)\right)^{-2} t^{-r} & \left(\left(b_{1}-t^{r-1}\left(1+t \beta^{\prime}(t)(\beta(t))^{-1}\right)\right)\left(y-c t^{r+1}\right)^{2}\right. \\
& \left.+\left(y-c t^{r+1}\right) \Lambda_{1}(t)\right)
\end{aligned}
$$


where

$$
\left|\Lambda_{1}(t)\right| \leqslant M t^{r+1} \beta(t)\left(q_{1}\left|b_{2}\right|+Q \frac{\left|b_{3}\right|}{q_{2}^{r-1}}+\frac{\left|\left(b_{3}-1\right) c\right| L_{1}+1}{M}+\omega_{1}(t)\right), \quad \lim _{t \rightarrow+0} \omega_{1}(t)=0 .
$$

There exists a (small enough) $\rho_{2}>0$ (see our choice of $M$ at the beginning of the proof) such that if $\rho<\rho_{2}$, then

$$
\left|\Lambda_{1}(t)\right|<M t^{r+1} \beta(t)\left|b_{1}\right|, \quad t \in(0, \rho] .
$$

Since $M t^{r+1} \beta(t)=\left|y-c t^{r+1}\right|$ when $(t, y) \in \Phi_{1}$, it is easy to see that

$$
\operatorname{sign} a_{1}(t, y)=\operatorname{sign} b_{1} \quad \text { when }(t, y) \in \Phi_{1} .
$$

(1) Let $b_{1}>0$ and therefore $a_{1}(t, y)>0$ when $(t, y) \in \Phi_{1}$. We prove that the integral curve of (2.17) which intersects $\Phi_{1}$ at an arbitrary point $\left(t_{0}, y_{0}\right)$ for sufficiently small $\left|t-t_{0}\right|(t \leqslant \rho)$ lies in $\mathscr{D}_{1}$ if $t<t_{0}$ and lies outside of $\overline{\mathscr{D}_{1}}$ if $t>t_{0}$. In fact, let $P\left(t_{0}, x_{0}\right)$ be an arbitrary point belonging to $\Phi_{1}$ and let $J_{P}:\left(t, y_{P}(t)\right)$ be the integral curve of (2.17) which passes through the point $P$. Then

$$
A_{1}\left(t_{0}, y_{P}\left(t_{0}\right)\right)=M^{2}, \quad a_{1}\left(t_{0}, y_{P}\left(t_{0}\right)\right)>0 .
$$

Therefore, if $t_{0} \in(0, \rho)$, then there exists $\delta>0$ such that

$$
\operatorname{sign}\left(A_{1}\left(t, y_{P}(t)\right)-A_{1}\left(t_{0}, y_{P}\left(t_{0}\right)\right)\right)=\operatorname{sign}\left(t-t_{0}\right), \quad\left|t-t_{0}\right|<\delta,
$$

or

$$
\operatorname{sign}\left(\left|y_{P}(t)-c t^{r+1}\right|\left(t^{r+1} \beta(t)\right)^{-1}-M\right)=\operatorname{sign}\left(t-t_{0}\right), \quad\left|t-t_{0}\right|<\delta .
$$

But this means that $\left(t, y_{P}(t)\right)$ lies in $\mathscr{D}_{1}$ if $t \in\left(t_{0}-\delta, t_{0}\right)$ and $\left(t, y_{P}(t)\right)$ lies outside of $\overline{\mathscr{D}_{1}}$ if $t \in\left(t_{0}, t_{0}+\delta\right)$. If $t_{0}=\rho$, then there exists $\delta>0$ such that

$$
A_{1}\left(t, y_{P}(t)\right)<A_{1}\left(\rho, y_{P}(\rho)\right), \quad t \in(\rho-\delta, \rho)
$$

or

$$
\left|y_{P}(t)-c t^{r+1}\right|\left(t^{r+1} \beta(t)\right)^{-1}<M, \quad t \in(\rho-\delta, \rho),
$$

and this means that $\left(t, y_{P}(t)\right)$ lies in $\mathscr{D}_{1}, t \in(\rho-\delta, \rho)$. It follows from this that each of the integral curves of (2.17) which intersect $H$ remains in $\mathscr{D}_{1}$ for all $t \in(0, \rho]$. We consider an arbitrary point $G\left(\rho, y_{G}\right) \in H$ and let $J_{u}:\left(t, y_{u}(t)\right)$ be the integral curve of (2.17) such that $y_{u}(\rho)=y_{G}$. It is easy to see that

$$
\begin{gathered}
\left|y_{u}(t)-c t^{r+1}\right| \leqslant M t^{r+1} \beta(t), \quad t \in(0, \rho], \\
\left|y_{u}^{\prime}(t)-(r+1) c t^{r}\right| \leqslant\left(2\left|b_{1}\right|+\omega_{2}(t)\right) M t \beta(t), \quad \lim _{t \rightarrow+0} \omega_{2}(t)=0 .
\end{gathered}
$$


There exists a (small enough) $\rho_{3}>0$ such that if $\rho<\rho_{3}$ (see our choice of $Q$ at the beginning of the proof), then

$$
\left|y_{u}^{\prime}(t)-(r+1) c t^{r}\right| \leqslant Q M t \beta(t), \quad t \in(0, \rho] .
$$

Now we set

$$
y_{u}(0)=0, \quad y_{u}^{\prime}(0)=0 .
$$

Now we will prove that condition (2.13) is fulfilled. Let $\varepsilon>0$ be given. We consider arbitrary points $t_{i} \in[0, \rho], i \in\{1,2\}$. We have the following.

(a) If $t_{i} \in\left[0, t_{\varepsilon}\right], i \in\{1,2\}$, then, in view of (2.14), (2.33),

$$
\begin{aligned}
\left|y_{u}^{\prime}\left(t_{1}\right)-y_{u}^{\prime}\left(t_{2}\right)\right| \leqslant & \left|y_{u}^{\prime}\left(t_{1}\right)-(1+r) c t_{1}^{r}\right|+(1+r)|c| t_{1}^{r}+\left|y_{u}^{\prime}\left(t_{2}\right)-(1+r) c t_{2}^{r}\right| \\
& +(1+r)|c| t_{2}^{r} \leqslant Q M t_{1} \beta\left(t_{1}\right)+(1+r)|c| t_{1}^{r} \\
& +Q M t_{2} \beta\left(t_{2}\right)+(1+r)|c| t_{2}^{r} \leqslant \frac{4}{33} \varepsilon<\frac{\varepsilon}{8}<\varepsilon .
\end{aligned}
$$

(b) If $t_{i} \in\left[t_{\varepsilon}, \rho\right], i \in\{1,2\}$, and $\left|t_{1}-t_{2}\right| \leqslant \delta(\varepsilon)$, then it is easy to see that there exists a (small enough) $\rho_{4}>0$ such that if $\rho<\rho_{4}$, then

$$
\begin{aligned}
\left|y_{u}^{\prime}\left(t_{1}\right)-y_{u}^{\prime}\left(t_{2}\right)\right| \leqslant & l_{2}\left(t_{1}\right)\left|u^{\prime}\left(t_{1}\right)-u^{\prime}\left(t_{2}\right)\right| \\
& +\left(\left|b_{3}\right|+l_{2}\left(t_{1}\right)\right) q_{2}^{-r}\left|u^{\prime}\left(q_{2} t_{1}\right)-u^{\prime}\left(q_{2} t_{2}\right)\right|+B\left(t_{\varepsilon}\right)\left|t_{1}-t_{2}\right| .
\end{aligned}
$$

Moreover, there exists a (small enough) $\rho_{5}>0$ such that if $\rho<\rho_{5}$, then

$$
l_{2}(t) \leqslant \frac{1}{8}, \quad l_{2}(t) \leqslant \frac{q_{2}^{r}}{8}, \quad t \in(0, \rho] .
$$

In addition to this, it follows from (2.6) that

$$
\left|b_{3}\right| q_{2}^{-r}<\frac{1}{2}
$$

Therefore,

$$
\begin{aligned}
\left|y_{u}^{\prime}\left(t_{1}\right)-y_{u}^{\prime}\left(t_{2}\right)\right| \leqslant & \frac{1}{8}\left|u^{\prime}\left(t_{1}\right)-u^{\prime}\left(t_{2}\right)\right| \\
& +\frac{5}{8}\left|u^{\prime}\left(q_{2} t_{1}\right)-u^{\prime}\left(q_{2} t_{2}\right)\right|+B\left(t_{\varepsilon}\right)\left|t_{1}-t_{2}\right| .
\end{aligned}
$$

By assumption, $\left|u^{\prime}\left(t_{1}\right)-u^{\prime}\left(t_{2}\right)\right| \leqslant \varepsilon$. Moreover, since $q_{2} t_{i} \in[0, \rho], i \in\{1,2\}$, and

$$
\left|q_{2} t_{1}-q_{2} t_{2}\right|=q_{2}\left|t_{1}-t_{2}\right| \leqslant q_{2} \delta(\varepsilon) \leqslant \delta(\varepsilon),
$$

we have, at the same time, $\left|u^{\prime}\left(q_{2} t_{1}\right)-u^{\prime}\left(q_{2} t_{2}\right)\right| \leqslant \varepsilon$. Thus,

$$
\left|y_{u}^{\prime}\left(t_{1}\right)-y_{u}^{\prime}\left(t_{2}\right)\right| \leqslant \frac{\varepsilon}{8}+\frac{5 \varepsilon}{8}+B\left(t_{\varepsilon}\right) \delta(\varepsilon)=\frac{7 \varepsilon}{8}<\varepsilon .
$$


(c) If $t_{1} \in\left[0, t_{\varepsilon}\right], t_{2} \in\left[t_{\varepsilon}, \rho\right]$, or vice versa, and $\left|t_{1}-t_{2}\right| \leqslant \delta(\varepsilon)$, then we have $t_{1}, t_{\varepsilon}$ belonging to $\left[0, t_{\varepsilon}\right], t_{\varepsilon}, t_{2}$ belonging to $\left[t_{\varepsilon}, \rho\right]$, and $\left|t_{\varepsilon}-t_{2}\right| \leqslant\left|t_{1}-t_{2}\right| \leqslant \delta(\varepsilon)$.

Therefore, in view of (2.35) and (2.41),

$$
\left|y_{u}^{\prime}\left(t_{1}\right)-y_{u}^{\prime}\left(t_{2}\right)\right| \leqslant\left|y_{u}^{\prime}\left(t_{1}\right)-y_{u}^{\prime}\left(t_{\varepsilon}\right)\right|+\left|y_{u}^{\prime}\left(t_{\varepsilon}\right)-y_{u}^{\prime}\left(t_{2}\right)\right|<\frac{\varepsilon}{8}+\frac{7 \varepsilon}{8}=\varepsilon .
$$

This completes the proof of $y_{u} \in \mathcal{U}$.

(2) Let $b_{1}<0$ and therefore $a_{1}(t, y)<0$ when $(t, y) \in \Phi_{1}$. Similarly, as in the case $b_{1}>0$, it can be proved that if an integral curve of (2.17) intersects $\Phi_{1}$ at an arbitrary point $\left(t_{0}, y_{0}\right)$, then for sufficiently small $\left|t-t_{0}\right|(t \leqslant \rho)$, the curve indicated lies in $\mathscr{D}_{1}$ if $t>t_{0}$ and it lies outside of $\overline{\mathscr{D}_{1}}$ if $t<t_{0}$. It follows that at least one of the integral curves of (2.17) which intersect $H$ is defined for all $t \in(0, \rho]$ and lies in $\mathscr{D}_{1}$ if $t \in(0, \rho]$. In fact, if an integral curve of (2.17) intersects $\Phi_{1}$, then it has to intersect $\bar{H}$. Let the mapping $\psi: \Phi_{1} \rightarrow$ $\bar{H}$ be defined in the following way: the point $\psi(P) \in \bar{H}$ is assigned to the point $P \in \Phi_{1}$ if both of these points belong to a common integral curve of $(2.17)$. We denote $\psi\left(\Phi_{1}\right)=$ $\left\{\psi(P): P \in \Phi_{1}\right\}$. The set $\bar{H} \backslash \psi\left(\Phi_{1}\right)$ is a nonempty one $\left(\bar{H}\right.$ is a closed set, but $\psi\left(\Phi_{1}\right)$ is a nonclosed set since $\psi\left(\Phi_{1}\right)$ is the image of the nonclosed set $\left.\Phi_{1}\right)$. Let $J_{u}:\left(t, y_{u}(t)\right)$ be an integral curve of (2.17) such that $\left(\rho, y_{u}(\rho)\right) \in \bar{H} \backslash \psi\left(\Phi_{1}\right)$. Then $J_{u}:\left(t, y_{u}(t)\right)$ cannot have common points with $\Phi_{1}$. Therefore, $J_{u}:\left(t, y_{u}(t)\right)$ is defined for all $t \in(0, \rho]$ and it lies in $\mathscr{D}_{1}$ if $t \in(0, \rho]$. As in the case $b_{1}>0$, we can prove that the inequalities $(2.31),(2.33)$ are fulfilled, and we can introduce notation (2.34) and prove that condition (2.13) is fulfilled. Thus, $y_{u} \in \mathcal{U}$.

Now we will prove in the case $b_{1}<0$ that if $t \rightarrow+0$, then all integral curves of (2.17) leave the set $\overline{\mathscr{D}_{1}} \backslash\{(0,0)\}$, with one exception, $J_{u}:\left(t, y_{u}(t)\right)$. Let $b_{1}<0$; we denote

$$
\begin{aligned}
& \Phi_{2}(v)=\left\{(t, y): t \in(0, \rho],\left|y-y_{u}(t)\right|=v t^{r+1} \beta(t)(-\ln t)\right\}, \\
& \mathscr{D}_{2}(v)=\left\{(t, y): t \in(0, \rho],\left|y-y_{u}(t)\right|<v t^{r+1} \beta(t)(-\ln t)\right\},
\end{aligned}
$$

where $v$ is a parameter, $v \in(0,1]$. Let the function $A_{2}: \mathscr{D}_{0} \rightarrow[0,+\infty)$ be defined by the equality

$$
A_{2}(t, y)=\left(y-y_{u}(t)\right)^{2}\left(t^{r+1} \beta(t)(-\ln t)\right)^{-2}
$$

and let $a_{2}: \mathscr{D}_{0} \rightarrow \mathbb{R}$ be the derivative of the function $A_{2}$. Using (2.17), we have

$$
a_{2}(t, y)=2\left(t^{r+1} \beta(t)(-\ln t)\right)^{-2} t^{-r}\left(y-y_{u}(t)\right)^{2}\left(b_{1}-t^{r-1}\left(1+t \beta^{\prime}(t)(\beta(t))^{-1}+(\ln t)^{-1}\right)\right) .
$$

There exists a (small enough) $\rho_{6}>0$ such that if $\rho<\rho_{6}$, then $a_{2}(t, y)<0$ when $(t, y) \in$ $\mathscr{D}_{0}, y \neq y_{u}(t)$. In particular, $a_{2}(t, y)<0$ when $(t, y) \in \Phi_{2}(v)$ for any $v \in(0,1]$. Therefore, for any $\nu \in(0,1]$, an integral curve of (2.17) which intersects $\Phi_{2}(\nu)$ in an arbitrary point $\left(t_{0}, y_{0}\right)$, for sufficiently small $\left|t-t_{0}\right|(t \leqslant \rho)$, lies in $\mathscr{D}_{2}(\nu)$ if $t>t_{0}$ and lies outside of $\overline{\mathscr{D}_{2}(\nu)}$ if $t<t_{0}$ (the proof is similar to the one for $\left.\Phi_{1}\right)$. Let $P_{*}\left(t_{*}, y_{*}\right) \in \overline{\mathscr{D}_{1}} \backslash\{(0,0)\}, y_{*} \neq y_{u}\left(t_{*}\right)$. Then there exists $v_{*} \in(0,1]$ such that $P_{*} \in \Phi_{2}\left(\nu_{*}\right)$. As above, the integral curve of $(2.17)$ $J_{*}:\left(t, y_{*}(t)\right)$, which passes through $P_{*}$, lies outside of $\overline{\mathscr{D}_{2}\left(\nu_{*}\right)}$ if $t \in\left(t_{-}, t_{*}\right)$, where $\left(t_{-}, t_{*}\right)$ 
is the left maximal existence interval for the solution $y_{*}$. On the other hand, if $(t, y) \in$ $\overline{\mathscr{D}_{1}} \backslash\{(0,0)\}$, then

$$
\left|y-y_{u}(t)\right| \leqslant\left|y-c t^{r+1}\right|+\left|y_{u}(t)-c t^{r+1}\right| \leqslant 2 M t^{r+1} \beta(t)<v_{*} t^{r+1} \beta(t)(-\ln t)
$$

if $t \in\left(0, t_{* *}\right)$, where $t_{* *} \in(0, \rho)$ and $t_{* *}$ is small enough. Thus, if $(t, y) \in \overline{\mathscr{D}_{1}} \backslash\{(0,0)\}$ and $t \in\left(0, t_{* *}\right)$, then $(t, y) \in \mathscr{D}_{2}\left(\nu_{*}\right)$. Let $t^{*}=\min \left\{t_{*}, t_{* *}\right\}$. As above, the integral curve $J_{*}:\left(t, y_{*}(t)\right)$ lies outside of $\overline{\mathscr{D}_{1}} \backslash\{(0,0)\}$ if $t \in\left(t_{-}, t^{*}\right)$ and we have proved our statement.

Now we can introduce an operator $\mathrm{T}: \boldsymbol{U} \rightarrow \boldsymbol{U}$ by $(\mathrm{T} u)(t)=y_{u}(t)$. We prove that $\mathrm{T}:$ $\mathcal{U} \rightarrow \boldsymbol{U}$ is a continuous operator. Let $u_{i} \in \mathcal{U}, i \in\{1,2\}$, be arbitrary functions and let $\mathrm{T} u_{i}=y_{i}, i \in\{1,2\}$. If $u_{1}=u_{2}$, then $y_{1}=y_{2}$. Let $\left\|u_{1}-u_{2}\right\|_{\mathscr{B}}=d, d>0$. We will investigate the behavior of the integral curves of the differential equation

$$
\begin{aligned}
y^{\prime}(t)= & a t+b_{1} y(t) t^{-r}+r y(t) t^{-1}+b_{2} u_{1}\left(q_{1} t\right)\left(q_{1} t\right)^{-r} \\
& +b_{3} q_{2}^{-r} u_{1}^{\prime}\left(q_{2} t\right)-r b_{3} q_{2}^{-r-1} t^{-1} u_{1}\left(q_{2} t\right) \\
& +\varphi\left(t, u_{1}(t) t^{-r}, u_{1}\left(q_{1} t\right)\left(q_{1} t\right)^{-r}, u_{1}^{\prime}(t) t^{-r}\right. \\
& \left.\quad-r u_{1}(t) t^{-r-1}, u_{1}^{\prime}\left(q_{2} t\right)\left(q_{2} t\right)^{-r}-r u_{1}\left(q_{2} t\right)\left(q_{2} t\right)^{-r-1}\right) .
\end{aligned}
$$

(It is obvious that $y_{1}:(0, \rho] \rightarrow \mathbb{R}$ is the solution of (2.47).) We introduce the following notations:

$$
\begin{aligned}
& \Phi_{3}=\left\{(t, y): t \in(0, \rho],\left|y-y_{2}(t)\right|=\gamma d^{\nu}\left(t^{r+1} \beta(t)\right)^{1-\gamma}\right\}, \\
& \mathscr{D}_{3}=\left\{(t, y): t \in(0, \rho],\left|y-y_{2}(t)\right|<\gamma d^{\nu}\left(t^{r+1} \beta(t)\right)^{1-\nu}\right\},
\end{aligned}
$$

where $v=(r+2)^{-1}, \gamma=3\left|b_{1}\right|^{-1}(2 M)^{1-\nu}\left(\left|b_{2}\right|+1\right)+1$.

Let the function $A_{3}: \mathscr{D}_{0} \rightarrow[0,+\infty)$ be defined by the equality

$$
A_{3}(t, y)=\left(y-y_{2}(t)\right)^{2}\left(t^{r+1} \beta(t)\right)^{-2(1-v)}
$$

and let $a_{3}: \mathscr{D}_{0} \rightarrow \mathbb{R}$ be the derivative of the function $A_{3}$. Using (2.47), we have

$$
\begin{aligned}
a_{3}(t, y)=2\left(t^{1+r} \beta(t)\right)^{-2(1-\nu)} t^{-r}( & \left(b_{1}+(-1+\nu(1+r)\right. \\
& \left.\left.-(1-\nu) t \beta^{\prime}(t)(\beta(t))^{-1}\right) t^{r-1}\right)\left(y-y_{2}(t)\right)^{2} \\
+ & \left.\left(y-y_{2}(t)\right) \Lambda_{3}(t)\right),
\end{aligned}
$$

and there exists a (small enough) $\rho_{7}>0$ such that if $\rho<\rho_{7}$, then

$$
\begin{aligned}
\left|\Lambda_{3}(t)\right| & \leqslant(2 M)^{1-\nu}\left(\left|b_{2}\right|+1\right) d^{\nu}\left(t^{1+r} \beta(t)\right)^{1-\nu} \\
& <\frac{\left|b_{1}\right|}{3}\left|y-y_{2}(t)\right| \quad \text { when }(t, y) \in \Phi_{3},
\end{aligned}
$$


since

$$
\begin{aligned}
\left|u_{1}(t)-u_{2}(t)\right| & \leqslant d^{v}\left(2 M t^{1+r} \beta(t)\right)^{1-\nu}, \\
\left|u_{1}\left(q_{i} t\right)-u_{2}\left(q_{i} t\right)\right| & \leqslant d^{v}\left(2 M\left(q_{i} t\right)^{1+r} \beta\left(q_{i} t\right)\right)^{1-\nu}, \quad i \in\{1,2\}, \\
\left|u_{1}^{\prime}(t)-u_{2}^{\prime}(t)\right| & \leqslant d^{v}(2 Q M t \beta(t))^{1-\nu}, \\
\left|u_{1}^{\prime}\left(q_{2} t\right)-u_{2}^{\prime}\left(q_{2} t\right)\right| & \leqslant d^{v}\left(2 Q M q_{2} t \beta\left(q_{2} t\right)\right)^{1-v}, \quad t \in(0, \rho]
\end{aligned}
$$

and the constant $\gamma$ is large enough.

There exists a (small enough) $\rho_{8}>0$ such that if $\rho<\rho_{8}$, then

$$
\operatorname{sign} a_{3}(t, y)=\operatorname{sign} b_{1} \quad \text { when }(t, y) \in \Phi_{3} .
$$

(1) Let $b_{1}>0$ and therefore $a_{3}(t, y)>0$ when $(t, y) \in \Phi_{3}$. Then an integral curve of (2.47) which intersects $\Phi_{3}$ in an arbitrary point $\left(t_{0}, y_{0}\right)$, for sufficiently small $\left|t-t_{0}\right|$ $(t \leqslant \rho)$, lies in $\mathscr{D}_{3}$ if $t<t_{0}$ and lies outside of $\overline{\mathscr{D}_{3}}$ if $t>t_{0}$. (The proof is similar to the one for $\Phi_{1}$ in the case $b_{1}>0$.) In addition, $y_{1}(\rho)=y_{2}(\rho)=y_{G}$. Thus, if $t$ decreases from $t=\rho$ to $t=0$, then the integral curve of $(2.47), J:\left(t, y_{1}(t)\right)$, lies in $\mathscr{D}_{3}$ for all $t \in(0, \rho]$ because this integral curve cannot intersect $\Phi_{3}$. We have

$$
\begin{aligned}
& \left|y_{1}(t)-y_{2}(t)\right| \leqslant \gamma d^{\nu}\left(t^{r+1} \beta(t)\right)^{1-\nu}, \quad t \in(0, \rho], \\
& \left|y_{1}^{\prime}(t)-y_{2}^{\prime}(t)\right| \leqslant\left(\left(\left|b_{1}\right|+r t^{r-1}\right) \gamma+(2 M)^{1-\nu}\left(\left|b_{2}\right|+1\right)\right) t^{(1+r)(1-v)-r}(\beta(t))^{1-\nu} d^{\nu} .
\end{aligned}
$$

Here $1-v>0,(1+r)(1-v)-r=(r+2)^{-1}>0$. Thus, there exists a (small enough) $\rho_{9}>0$ such that if $\rho<\rho_{9}$, then

$$
\left|y_{1}(t)-y_{2}(t)\right|+\left|y_{1}^{\prime}(t)-y_{2}^{\prime}(t)\right| \leqslant d^{\nu}, \quad t \in(0, \rho] .
$$

(2) Let $b_{1}<0$ and therefore $a_{3}(t, y)<0$ when $(t, y) \in \Phi_{3}$. Then an integral curve of (2.47) which intersects $\Phi_{3}$ in an arbitrary point $\left(t_{0}, y_{0}\right)$, for sufficiently small $\left|t-t_{0}\right|$ $(t \leqslant \rho)$, lies in $\mathscr{D}_{3}$ if $t>t_{0}$ and lies outside of $\overline{\mathscr{D}_{3}}$ if $t<t_{0}$. (The proof is similar to the one for $\Phi_{1}$ in the case $b_{1}<0$.) In addition,

$$
\left|y_{1}(t)-y_{2}(t)\right| \leqslant\left|y_{1}(t)-c t^{r+1}\right|+\left|y_{2}(t)-c t^{r+1}\right| \leqslant 2 M t^{r+1} \beta(t)<\gamma d^{v}\left(t^{r+1} \beta(t)\right)^{1-v}
$$

when $t \in(0, t(d)]$, where $t(d) \in(0, \rho)$ is small enough. Therefore, if $t \in(0, t(d)]$, then the integral curve of (2.47), $J:\left(t, y_{1}(t)\right)$, lies in $\mathscr{D}_{3}$. As above, if $t$ increases from $t=t(d)$ to $t=\rho$, then the integral curve of $(2.47), J:\left(t, y_{1}(t)\right)$, cannot intersect $\Phi_{3}$ and therefore this curve lies in $\mathscr{D}_{3}$ for all $t \in(0, \rho]$. Further, we can get (2.54) and (2.55) as in the case $b_{1}>0$.

Thus, if $\left\|u_{1}-u_{2}\right\|_{\mathscr{B}}=d<\delta(\varepsilon)=(\varepsilon / 2)^{1 / \nu}$, then

$$
\begin{aligned}
\max _{t \in[0, \rho]}\left(\left|y_{1}(t)-y_{2}(t)\right|+\left|y_{1}^{\prime}(t)-y_{2}^{\prime}(t)\right|\right) \\
=\left\|y_{1}-y_{2}\right\|_{\Re}=\left\|\mathrm{T} u_{1}-\mathrm{T} u_{2}\right\|_{\Re} \leqslant \frac{\varepsilon}{2}<\varepsilon .
\end{aligned}
$$


The reasoning given above does not depend on the choice of $\varepsilon>0$ and $u_{i} \in \mathcal{U}, i \in$ $\{1,2\}$. Therefore $T: U \rightarrow U$ is the continuous operator. To complete the proof of the theorem, it suffices to apply the Schauder fixed point theorem to the operator $\mathrm{T}: \boldsymbol{U} \rightarrow \boldsymbol{U}$.

\section{References}

[1] B. P. Demidovič, Lectures on the Mathematical Theory of Stability, Izdat. "Nauka", Moscow, 1967.

[2] L. J. Grimm, Analytic solutions of a neutral differential equation near a singular point, Proc. Amer. Math. Soc. 36 (1972), no. 1, 187-190.

[3] L. J. Grimm and L. M. Hall, Holomorphic solutions of singular functional differential equations, J. Math. Anal. Appl. 50 (1975), no. 3, 627-638.

[4] P. Hartman, Ordinary Differential Equations, John Wiley \& Sons, New York, 1964.

[5] A. I. Shindyapin, A boundary value problem for a singular equation, Differ. Uravn. 20 (1984), no. 3, 450-455 (Russian).

[6] D. R. Smart, Fixed Point Theorems, Cambridge University Press, London, 1974.

[7] A. E. Zernov, On the solvability and asymptotics of the solutions of a functional-differential equation with a singularity, Ukrain. Mat. Zh. 53 (2001), no. 4, 455-465 (Russian).

[8] Qualitative analysis of the implicit singular Cauchy problem, Ukrain. Mat. Zh. 53 (2001), no. 3, 302-310 (Russian).

Ravi P. Agarwal: Department of Mathematical Sciences, Florida Institute of Technology, Melbourne, FL 32901, USA

E-mail address: agarwal@fit.edu

Donal O’Regan: Department of Mathematics, National University of Ireland, Galway, Ireland E-mail address: donal.oregan@nuigalway.ie

Oleksandr E. Zernov: South Ukrainian State Pedagogical University, Odessa, Ukraine 


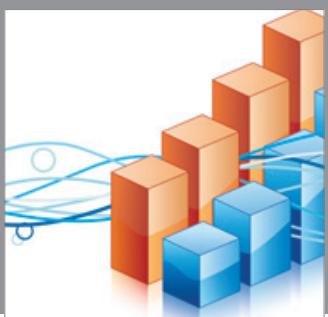

Advances in

Operations Research

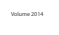

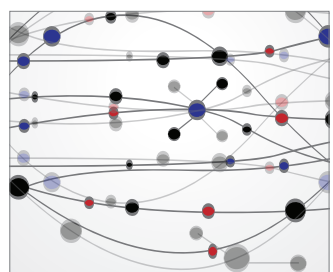

\section{The Scientific} World Journal
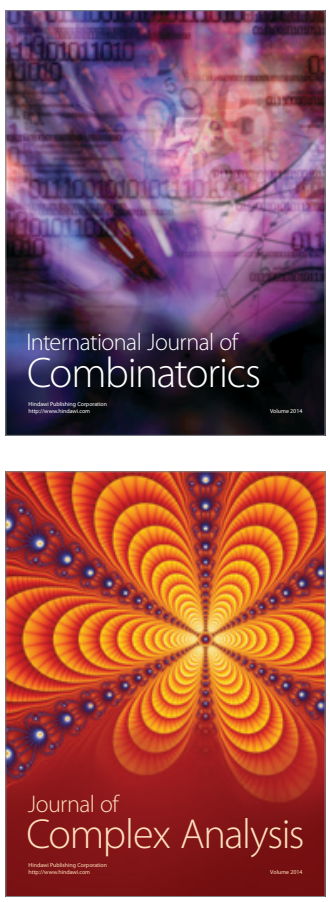

International Journal of

Mathematics and

Mathematical

Sciences
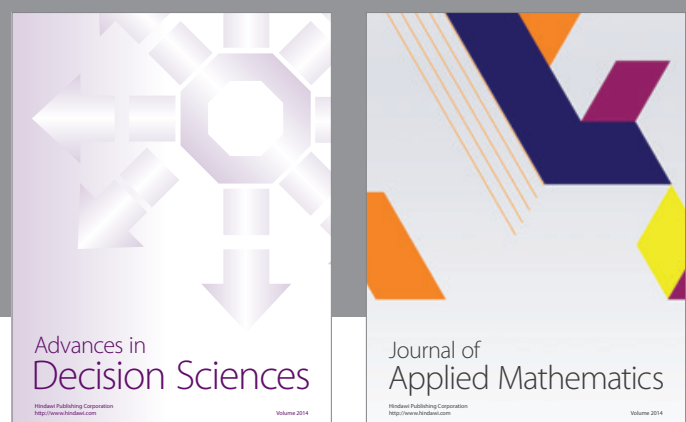

Journal of

Applied Mathematics
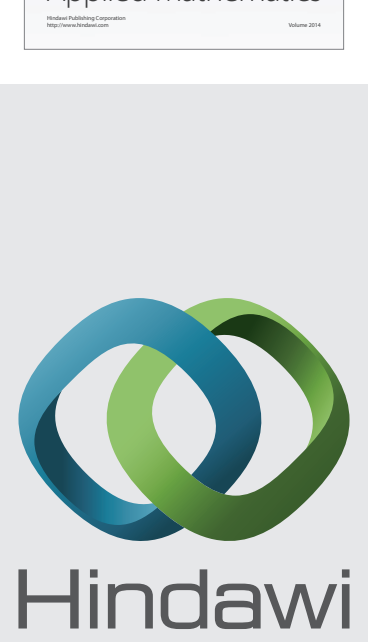

Submit your manuscripts at http://www.hindawi.com
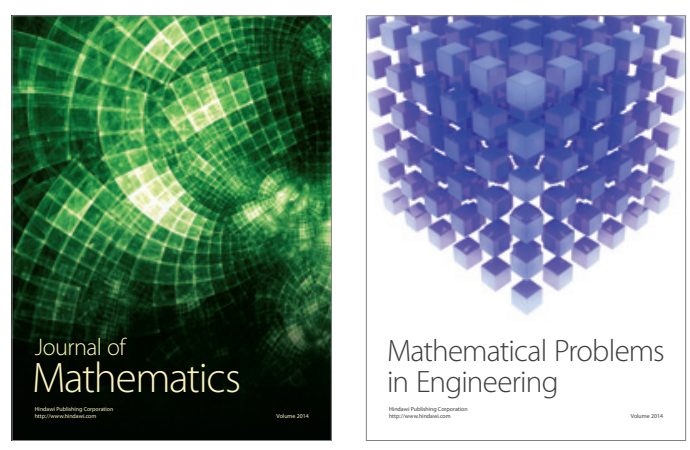

Mathematical Problems in Engineering
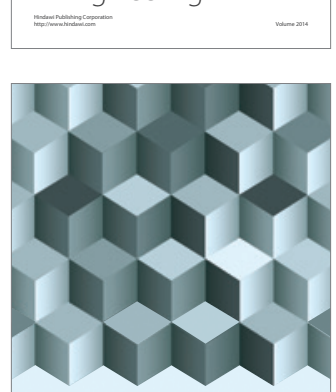

Journal of

Function Spaces
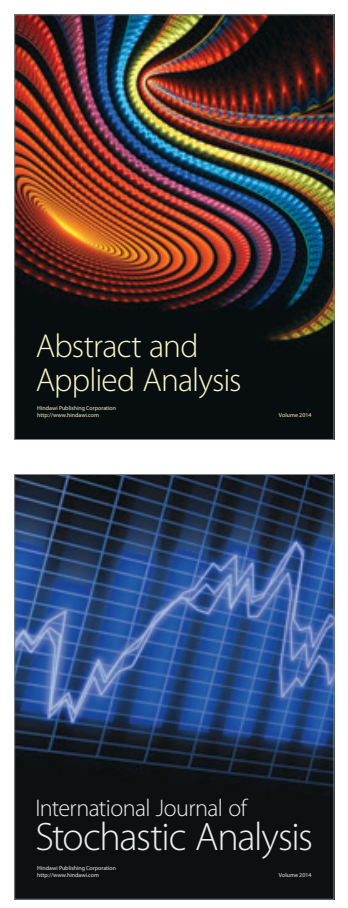

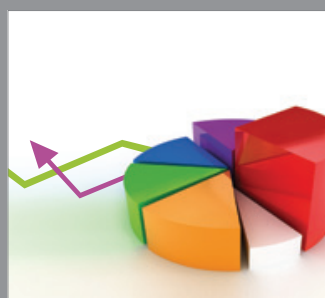

ournal of

Probability and Statistics

Promensencen
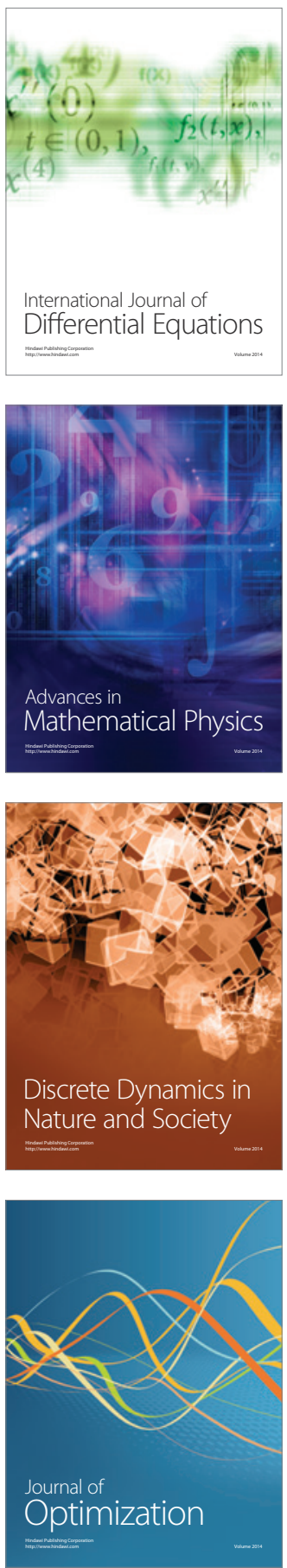